\title{
ON FUNCTIONS POSITIVE DEFINITE RELATIVE TO THE ORTHOGONAL GROUP AND THE REPRESENTATION OF FUNCTIONS AS HANKEL-STIELT JES TRANSFORMS
}

BY

\section{A. EDWARD NUSSBAUM( $\left.{ }^{1}\right)$}

ABSTRACT. To every continuous function $f$ on an interval $0 \leq x<a(0<a$ $\leq \infty)$ and every positive number $\nu$ associate the kernel

$$
f(x, y)=\int_{0}^{\pi} f\left(\left(x^{2}+y^{2}-2 x y \cos \theta\right)^{1 / 2}\right)(\sin \theta)^{2 \nu-1} d \theta, \quad 0<x, y<a / 2 .
$$

Let $\Omega(z)=\Gamma(\nu+1 / 2)(2 / z)^{\nu-1 / 2} J_{\nu-1 / 2}(z)$, where $J_{\nu-1 / 2}(z)$ is the Bessel function of index $\nu-1 / 2$. It is shown that $f$ has an integral representation $f(x)=$ $\int_{-\infty}^{\infty} \Omega(x \sqrt{\lambda}) d \gamma(\lambda)$, where $\gamma$ is a finite, positive Radon measure on $R$, if and only if the kernel $f(x, y)$ is positive definite. If $\nu=(N-1) / 2$, where $N$ is an integer $\geq 2$, this condition is equivalent to $f_{N}(x)=f(|x|), x \in R^{N},|x|<a$, is positive definite relative to the orthogonal group $O(N)$. The results of this investigation extend the preceding one of the author on functions positive definite relative to the orthogonal group. In particular they yield the result of Rudin on the extensions of radial positive definite functions.

1. Introduction. Let $f$ be a function defined on an open ball $S_{N}(a)=$ $\left\{x \in R^{N}|x|<a\right\}$ in $N$-dimensional Euclidean space $R^{N}$. Here $0<a \leq \infty$ and $|x|$ denotes the usual norm in $R^{N}$. In [4] we called $f$ positive definite relative to the orthogonal group $O(N)$ if $f$ satisfies the following conditions:

$f$ is continuous, radial and

$$
\iint f(x-y) \phi(x) \overline{\phi(y)} d x d y \geq 0
$$

for all radial $\phi$ in $C_{0}^{\infty}\left(S_{N}(a / 2)\right) \cdot\left(C_{0}^{\infty}(G)\right.$ denotes the vector space of complex valued infinitely differentiable functions on $G$ with compact support.)

In [4] we did show that a function $f$ defined on $S_{N}(a)$ is positive definite relative to $O(N)$ if and only if it has an integral representation of the form

$$
f(x)=\int_{R} e^{i x \cdot t} d \mu_{1}(t)+\int_{R^{N}} e^{x \cdot t} d \mu_{2}(t) \text { for all } x \in S_{N}(a),
$$

where $\mu_{1}$ and $\mu_{2}$ are bounded, positive, rotation invariant Radon measures on $R^{N}$ and $\mu_{2}$ is such that

Received by the editors September 24, 1971.

AMS (MOS) subject classifications (1970). Primary 42A88, 42A76, 43A35; Secondary 44A15.

Key words and phrases. Positive definite functions, integral representation, HankelStieltjes transform.

(1) This work was in part supported by National Science Foundation Grant NSF GP-19588. 


$$
\int_{R^{N}} e^{x \cdot t} d \mu_{2}(t)<\infty \text { for all } x \in S_{N}(a) \text {. }
$$

The integral representation (2) is equivalent [4, Theorem 3] to

$$
f(x)=\int_{-\infty}^{\infty} \Omega_{N}(|x| \sqrt{\lambda}) d y(\lambda) \text { for all } x \in S_{N}(a),
$$

where $\gamma$ is a bounded, positive Radon measure on $R$ and (for $\alpha>0$ )

$$
\begin{aligned}
\Omega_{a}(z) & =\Gamma\left(\frac{\alpha}{2}\right)\left(\frac{2}{z}\right)^{1 / 2(a-2)} J_{1 / 2(a-2)}(z) \quad \text { if } z \neq 0 \text { and } 1 \text { if } z=0 \\
& =1-\frac{z^{2}}{2 a}+\frac{z^{4}}{2 \cdot 4 a(a+2)}-\frac{z^{6}}{2 \cdot 4 \cdot 6 a(a+2)(a+4)}+\cdots,
\end{aligned}
$$

$J_{\nu}(z)$ is the Bessel function of order $\nu$.

The present investigation was inspired by the following observation.

Since the functions $f$ and $\phi$ in (1) are radial we may write $f(x)=F(|x|)$, $\phi(x)=\Phi(|x|)$, where $F$ is a function on $0 \leq r<a$ and $\Phi$ is a function on $0 \leq r<$ $a / 2$ with supp $\Phi \subset[0, a / 2)$ ( supp $\Phi$ denotes the support of $\Phi$ ). If we now introduce generalized spherical coordinates we find that for $N \geq 2$ inequality (1) becomes

(4) $\iint f(x-y) \phi(x) \overline{\phi(y)} d x d y=(2 \pi)^{N} \int_{0}^{a / 2} \int_{0}^{a / 2} f_{N}(r, s) \Phi(r) \overline{\Phi(s)} d \mu_{N}(r) d \mu_{N}(s) \geq 0$, where

$$
f_{N}(r, s)=\frac{1}{B[(N-1) / 2,1 / 2]} \int_{0}^{\pi} F\left(\sqrt{r^{2}+s^{2}-2 r s \cos \theta}\right)(\sin \theta)^{N-2} d \theta
$$

for $0 \leq r<a / 2,0 \leq s<a / 2$. Here $B[\alpha, \beta]=\Gamma(\alpha) \Gamma(\beta) / \Gamma(\alpha+\beta)$ is Euler's beta function and

$$
d \mu_{N}(r)=\left[2^{1 / 2(N-2)} \Gamma(N / 2)\right]^{-1} r^{N-1} d r .
$$

(4) remains valid also for $N=1$ if we set

$$
f_{1}(r, s)=1 / 2[F(|r+s|)+F(|r-s|)]
$$

for $0 \leq r<a / 2$ and $0 \leq s<a / 2$ (and $d \mu_{1}(r)$ as in (6)).

Indeed, if $N \geq 2$ and $x \in G=S_{N}(a / 2)$

$$
\int_{G} f(x-y) \overline{\phi(y)} d y=\int_{G} F(|x-y|) \overline{\Phi(|y|)} d y=\int_{0}^{a / 2} I(\rho) d \rho,
$$

where $I(\rho)$ is the surface integral of the function $F(|x-y|) \Phi(|y|),|y|=\rho$ over the sphere $\left\{y \in R^{N}|| y \mid=\rho\right\}$ of radius $\rho$ in $R^{N}$ :

$$
I(\rho)=\int_{|y|=\rho} F(|x-y|) \overline{\Phi(|y|)} d S .
$$

If we let $\theta$ be the angle between the vectors $x$ and $y$, then $|x-y|=$ $\sqrt{|x|^{2}+|y|^{2}-2|x||y| \cos \theta}$ and 


$$
d S=\frac{2 \pi^{(N-1) / 2}}{\Gamma((N-1) / 2)}(\rho \sin \theta)^{N-2} \rho d \theta
$$

( $d S$ is the infinitesimal $((n-1)$-dimensional) surface area of the spherical zone consisting of the points on the sphere $|y|=. \rho$ for which the angle between $y$ and $x$ varies from $\theta$ to $\theta+d \theta, d S$ is the product of $\rho d \theta$ with the $((n-2)$-dimensional $)$ area of the cross section of the sphere $|y|=\rho$ with the plane $(y, x)=$ $\sum_{i-1}^{N} y_{i} x_{i}=\rho \cos \theta$. The cross section is the surface of the sphere of radius $\rho \sin \theta$ in $R^{N-1}$; its area is

$$
\frac{2 \pi^{(N-1) / 2}}{\Gamma((N-1) / 2)}(\rho \sin \theta)^{N-2}
$$

Hence

$$
\left.d S=\frac{2 \pi^{(N-1) / 2}}{\Gamma((N-1) / 2)}(\rho \sin \theta)^{N-2} \rho d \theta .\right)
$$

From (10) and (9) follows that

(11) $I(\rho)=\frac{2 \pi^{(N-1) / 2}}{\Gamma((N-1) / 2)} \int_{0}^{\pi} F\left(\sqrt{|x|^{2}+\rho^{2}-2|x| \rho \cos \theta}\right)(\sin \theta)^{N-2} d \theta \cdot \overline{\Phi(\rho)} \rho^{N-1}$.

Now

$$
\frac{2 \pi^{(N-1) / 2}}{\Gamma((N-1) / 2)}=\frac{2 \pi^{N / 2}}{\Gamma((N-1) / 2) \Gamma(1 / 2)}=\frac{(2 \pi)^{N / 2}}{B[(N-1) / 2,1 / 2]}\left[2^{1 / 2(N-2)} \Gamma(N / 2)\right]^{-1}
$$

and hence, by (11) and (8),

$$
\begin{array}{r}
\int_{G} f(x-y) \overline{\phi(y)} d y \\
=\frac{(2 \pi)^{N / 2}}{B[(N-1) / 2,1 / 2]} \int_{0}^{a / 2} \int_{0}^{\pi} F\left(\sqrt{\left.|x|^{2}+\rho^{2}-2|x| \rho \cos \theta\right)}\right. \\
\cdot(\sin \theta)^{N-2} \Phi(\rho) d \theta d \mu_{N}(\rho) .
\end{array}
$$

Therefore

$$
\begin{aligned}
& \int_{G} \int_{G} f(x-y) \phi(x) \overline{\phi(y)} d x d y \\
& =\frac{(2 \pi)^{N}}{B[(N-1) / 2,1 / 2]} \int_{0}^{a / 2} \int_{0}^{a / 2} \int_{0}^{\pi} F\left(\sqrt{r^{2}+\rho^{2}-2 r \rho \cos \theta}\right) \\
& \text { - }(\sin \theta)^{N-2} \Phi(r) \overline{\Phi(\rho)} d \theta d \mu_{N}(r) d \mu_{N}(\rho),
\end{aligned}
$$

whence (4). The case $N=1$ is easily verified.

From (4) follows the following proposition

Proposition 1. Let $f(x)=F(|x|)$ be a continuous radial function on the ball $S_{N}(a)(0<a \leq \infty)$ in $R^{N}$ and set 


$$
F_{N}(r, s)=\int_{0}^{\pi} F\left(\sqrt{\left.r^{2}+s^{2}-2 r s \cos \theta\right)}(\sin \theta)^{N-2} d \theta\right.
$$

if $N \geq 2$ and

$$
F_{1}(r, s)=1 / 2[F(|r+s|)+F(|r-s|)]
$$

if $N=1$. Then $f$ is positive definite relative to the orthogonal group $O(N)$ if and only if the kernel $F_{N}(r, s), 0<r, s<a / 2$, is positive definite. That is,

$$
\sum_{i=1}^{n} \sum_{j=1}^{n} \alpha_{i} \bar{\alpha}_{j} F_{N}\left(x_{i}, x_{j}\right) \geq 0
$$

for all choices of complex numbers $\alpha_{1}, a_{2}, \cdots, \alpha_{n}$ and points $x_{1}, x_{2}, \cdots, x_{n}$ on the interval $(0, a / 2)$.

Proposition 1 allows us to rephrase the theorem [4, Theorem 31 on the integral representation (3) of a function positive definite relative to the orthogonal group $O(N)$ as follows.

Theorem 1. Let $F$ be a continuous function on the interval $0 \leq r<a$ $(0<a \leq \infty)$ and $N$ a positive integer. Let $F_{N}(r, s)$ be as in Proposition 1. Then $F$ bas an integral representation of the form

$$
F(r)=\int_{-\infty}^{\infty} \Omega_{N}(r \sqrt{\lambda}) d \gamma(\lambda) \text { for all } 0 \leq r<a,
$$

where $\gamma$ is a bounded, positive Radon measure on $R$, if and only if (13) bolds.

The principal purpose of this paper is to extend Theorem 1 (plus some refinements) to the case where $N=\alpha$ is any number $>1$. The method of proof is entirely parallel to the one used by the author in [4]. It is based on the "nuclear spectral theorem" [4, Theorem 1] used with great success by the Russian school. We assume that the reader is acquainted with this theorem. See loc. cit. The main theorem (Theorem 2) of this paper also extends the theorem in [1] in several ways. We were not aware at the time we obtained the result in [1] of the connection with functions positive definite relative to the orthogonal group. In conclusion we would like to point out that the main theorem of this paper also contains the result of Rudin [5] on the extension of radial positive definite functions. See the discussion in [4].

2. Definitions and preliminary results. Let $\nu$ be a fixed positive number. We define $\Omega(x)=\Omega_{2 \nu+1}(x)$, where $\Omega_{\alpha}(x)$ is as defined in the introduction.

We set

$$
d \mu(x)=\left[2^{\nu-1 / 2} \Gamma(\nu+1 / 2)\right]^{-1} x^{2 \nu} d x
$$

and define $L[1[0, \infty)$ as the linear space of Lebesgue measurable functions on 
$[0, \infty)$ for which $\int_{0}^{\infty}|f(x)| d \mu(x)<\infty$. Further we let

$$
D(x, y, z)=\frac{2^{(3 \nu-5 / 2)}[\Gamma(\nu+1 / 2)]^{2}}{\Gamma(\nu) \pi^{1 / 2}}(x y z)^{1-2 \nu}[A(x, y, z)]^{2 \nu-2},
$$

where $A(x, y, z)$ is the area of a triangle whose sides have length $x, y, z$ if there is such a triangle, and otherwise, $D(x, y, z)=0$.

Definition 1. If $f$ is a Lebesgue measurable locally essentially bounded function on the interval $[0, a)(0<a \leq \infty)$ we define the associated function or associated kernel $f(x, y)$ by

$$
f(x, y)=\frac{1}{c_{\nu}} \int_{0}^{\pi} f\left(\sqrt{x^{2}+y^{2}-2 x y \cos \theta}\right)(\sin \theta)^{2 \nu-1} d \theta
$$

for $0<x<a / 2,0<y<a / 2$. Here $c_{\nu}=B[\nu, 1 / 2]=\Gamma(\nu) \pi^{1 / 2} / \Gamma(\nu+1 / 2)$.

Proposition 2. If $f$ is as in Definition 1 and $f(x, y)$ its associated function, then

(i) $f(x, y)=\int_{0}^{a} f(z) D(x, y, z) d \mu(z)$ for $0<x<a / 2,0<y<a / 2$ and

(ii) $f(x, y)$ is continuous on $(0, a / 2) \times(0, a / 2)$.

Proof. Let $0<x<a / 2,0<y<a / 2$ and consider a triangle whose sides have length $x, y, z$ and whose angle opposite the side of length $z$ is $\theta$. Then $z=$ $\left(x^{2}+y^{2}-2 x y \cos \theta\right)^{1 / 2}$, whence $z d z=x y \sin \theta d \theta$. The area of the triangle $A(x, y, z)=x y \sin \theta / 2$. Therefore $d \theta=z d z / 2 A(x, y, z)$ and hence

$$
\begin{aligned}
& (\sin \theta)^{2 \nu-1} d \theta=\left(\frac{2 A(x, y, z)}{x y}\right)^{2 \nu-1} \frac{z}{2 A(x, y, z)} d z \\
& =2^{(2 \nu-2)}(x y z)^{1-2 \nu}[A(x, y, z)]^{2 \nu-2} z^{2 \nu} d z \text {. }
\end{aligned}
$$

Therefore

$$
\frac{1}{c_{\nu}}(\sin \theta)^{2 \nu-1} d \theta=D(x, y, z) d \mu(z)
$$

whence

$$
\begin{aligned}
\frac{1}{c_{\nu}} \int_{0}^{\pi} f\left(\sqrt{x^{2}+y^{2}-2 x y \cos \theta}\right)(\sin \theta)^{2 \nu-1} d \theta \\
=\int_{|x-y|}^{x+y} f(z) D(x, y, z) d \mu(z)=\int_{0}^{a} f(z) D(x, y, z) d \mu(z),
\end{aligned}
$$

for $D(x, y, z)=0$ if $z \geq x+y$ or $|x-y| \geq z$. This proves (i). In particular if we take $f(x) \equiv 1$ we obtain

$$
\int_{0}^{\infty} D(x, y, z) d \mu(z)=\frac{1}{c_{\nu}} \int_{0}^{\pi}(\sin \theta)^{2 \nu-1} d \theta=1, \quad 0<x, y<\infty
$$


for $B[\alpha, \beta]=2 \int_{0}^{\pi / 2}(\sin \theta)^{2 a-1}(\cos \theta)^{2 \beta-1} d \theta$. If now $0<x_{0}, y_{0}<a / 2$ and $0<$ $2 \delta<a-\left(x_{0}+y_{0}\right)$ then

$$
\begin{aligned}
& \left|f(x, y)-f\left(x_{0}, y_{0}\right)\right| \leq \int_{0}^{x_{0}+y_{0}+2 \delta}|f(z)|\left|D(x, y, z)-D\left(x_{0}, y_{0}, z\right)\right| d \mu(z) \\
& \leq \underset{0 \leq z_{\leq x}+x_{0}+2 \delta}{\operatorname{ess} \sup _{0}}|f(z)| \int_{0}^{\infty}\left|D(x, y, z)-D\left(x_{0}, y_{0}, z\right)\right| d \mu(z)
\end{aligned}
$$

for all $\left|x-x_{0}\right|<\delta,\left|y-y_{0}\right|<\delta$.

But

$$
\lim _{(x, y) \rightarrow\left(x_{0}, y_{0}\right)} \int_{0}^{\infty}\left|D(x, y, z)-D\left(x_{0}, y_{0}, z\right)\right| d \mu(z)=0
$$

since $D(x, y, z) \rightarrow D\left(x_{0}, y_{0}, z\right) \mu$-a.e. and

$$
\int_{0}^{\infty}|D(x, y, z)| d \mu(z)=1 \rightarrow \int_{0}^{\infty}\left|D\left(x_{0}, y_{0}, z\right)\right| d \mu(z)=1 \text {. }
$$

(We use here the fact that if $\left(f_{n}\right)$ is a sequence of integrable functions such that $f_{n} \rightarrow f$ a.e. with $f$ integrable, then $\int\left|f-f_{n}\right| \rightarrow 0$ if and only if $\int\left|f_{n}\right| \rightarrow \int|f|$.)

It follows that $f$ is continuous at $\left(x_{0}, y_{0}\right)$.

Lemma 1. If $f$ is a Lebesgue measurable locally essentially bounded function on $[0, a)(0<a \leq \infty)$ and its associated function $f(x, x)=0$ for $0<x<a / 2$, then $f(x)=0$ a.e. for $0 \leq x<a$.

\section{Proof.}

$$
f(x, x)=\frac{1}{c_{\nu}} \int_{0}^{\pi} f(x \sqrt{2-2 \cos \theta})(\sin \theta)^{2 \nu-1} d \theta=0 \text { for } 0<x<a / 2 .
$$

If we set, for fixed $0<x<a / 2, t=x \sqrt{2-2 \cos \theta} / 2$ we obtain

$$
f(x, x)=\frac{2^{2 \nu}}{c_{\nu} x^{4 \nu-2}} \int_{0}^{x} f(2 t) t^{2 \nu-1}\left(x^{2}-t^{2}\right)^{\nu-1} d t=0 .
$$

Hence

$$
\int_{0}^{x} f(2 t) t^{2 \nu-1}\left(x^{2}-t^{2}\right)^{\nu-1} d t=0, \quad 0<x<a / 2 .
$$

If $\nu=1, \int_{0}^{x} f(2 t) t d t=0$ for $0<x<a / 2$, whence $f(x)=0$ a.e. for $0 \leq x<a$.

If $\nu \neq 1$, we multiply both sides of equation (14) by $x$ and integrate $w$ ith respect to $x$ from 0 to $y$, we obtain

$$
\begin{aligned}
& \int_{0}^{y} \int_{0}^{x} f(2 t) t^{2 \nu-1} x\left(x^{2}-t^{2}\right)^{\nu-1} d t d x=\int_{0}^{y} \int_{t}^{y} f(2 t) t^{2 \nu-1} x\left(x^{2}-t^{2}\right)^{\nu-1} d x d t \\
& =\int_{0}^{y} f(2 t) t^{2 \nu-1} d t \int_{t}^{y} x\left(x^{2}-t^{2}\right)^{\nu-1} d x=\frac{1}{2 \nu} \int_{0}^{y} f(2 t) t^{2 \nu-1}\left(y^{2}-t^{2}\right)^{\nu} d t=0,
\end{aligned}
$$

for $0<y<a / 2$. Thus

$$
\int_{0}^{x} f(2 t) t^{2 \nu-1}\left(x^{2}-t^{2}\right)^{\nu} d t=0, \quad 0<x<a / 2 .
$$


Continuing this way we get

$$
\int_{0}^{x} f(2 t) t^{2 \nu-1}\left(x^{2}-t^{2}\right)^{\nu+k} d t=0 \text { for } 0<x<a / 2
$$

and $k=0,1,2, \ldots$, which upon the substitution $u=\sqrt{x^{2}-t^{2}}$ becomes

$$
\int_{0}^{x} f\left(2 \sqrt{x^{2}-u^{2}}\right)\left(x^{2}-u^{2}\right)^{\nu-1} u^{2 \nu+1} u^{2 k} d u=0
$$

for $0<x<a / 2$ and $k=0,1,2, \ldots$. Since every continuous function $g(u)$ on $[0, x]$ can be approximated uniformly by even polynomials in $u$, it follows from (16) that

$$
\int_{0}^{x} f\left(2 \sqrt{x^{2}-u^{2}}\right)\left(x^{2}-u^{2}\right)^{\nu-1} u^{2 \nu+1} g(u) d u=0
$$

for all continuous functions $g$ on $[0, x], 0<x<a / 2$. Therefore

$$
f\left(2 \sqrt{x^{2}-u^{2}}\right)\left(x^{2}-u^{2}\right)^{\nu-1} u^{2 \nu+1}=0 \text { a.e. for } 0 \leq u \leq x
$$

(for every fixed $0<x<a / 2$ ). Hence

$$
f\left(2 \sqrt{x^{2}-u^{2}}\right)=0 \text { a.e. for } 0 \leq u \leq x
$$

and every $0<x<a / 2$, whence $f(x)=0$ a.e. for $0 \leq x<a$.

If $f(x)=\Omega(\lambda x), 0 \leq x<\infty$, where $\lambda$ is any complex number, then its associated function

$$
f(x, y)=f(x) f(y), \quad 0<x, y<\infty .
$$

This formula is due to Sonine. See [8, formula (16), p. 367].

Thus by Proposition 2

$$
\int_{0}^{\infty} \Omega(\lambda z) D(x, y, z) d \mu(z)=\Omega(\lambda x) \Omega(\lambda y)
$$

for $0<x, y<\infty$ and all complex numbers $\lambda$.

If $f$ and $g$ are elements of $L_{\nu}^{1}[0, \infty)$, and if we set

$$
(f \times g)(x)=\int_{0}^{\infty} \int_{0}^{\infty} f(y) g(z) D(x, y, z) d \mu(y) d \mu(z),
$$

then $(f \times g)(x)$ exists for almost all $0<x<\infty, f \times g \in L_{\nu}^{1}[0, \infty)$, and (by Fubini's theorem and (17)) $(f \times g) \hat{\imath} \hat{f} \hat{g}$, where

$$
\hat{f}(t)=\int_{0}^{\infty} \Omega(x t) f(x) d \mu(x), \quad 0 \leq t<\infty,
$$

is the Hankel transform of $f$ of order $\nu-1 / 2$. See [2]. (Equation (12) states that if $\nu=(N-1) / 2$ then

$$
f * \bar{\phi}(x)=(2 \pi)^{N / 2} F \times \bar{\Phi}(|x|)
$$

This is the motivation for defining the " $x$-convolution" as above.) 
Definition 2. We denote by $P_{\nu}[0, a)(0<a \leq \infty)$ the set of Lebesgue measurable functions $f$ on the interval $[0, a)$ which are locally essentially bounded on $[0, a)$ and whose associated function $f(x, y)$ is positive definite on $(0, a / 2) \times$ $(0, a / 2)$. That is,

$$
\sum_{i=1}^{n} \sum_{j=1}^{n} \alpha_{i} \bar{\alpha}_{j} f\left(x_{i}, x_{j}\right) \geq 0
$$

for all choices of complex numbers $\alpha_{1}, \alpha_{2}, \ldots, \alpha_{n}$ and any points $x_{1}, x_{2}, \ldots, x_{n}$ on the interval $(0, a / 2)$. The elements of $P_{\nu}[0, a)$ will be called positive definite functions on $[0, a)$.

It is clear that condition (18) is equivalent with

$$
\int_{0}^{a / 2} \int_{0}^{a / 2} f(x, y) \phi(x) \overline{\phi(y)} d \mu(x) d \mu(y) \geq 0
$$

for all $\phi \in C_{0}[0, a / 2)$ (or all $\phi \in C_{0}^{\infty}(0, a / 2)$ ).

3. The principal result.

Theorem 2. A Lebesgue measurable function $f$ on the interval $[0, a)$ $(0<a \leq \infty)$ bas an integral representation of the form

$$
f(x)=\int_{-\infty}^{\infty} \Omega(x \sqrt{\lambda}) d y(\lambda), \text { a.e. for } 0 \leq x<a,
$$

where $\gamma$ is a bounded, positive Radon measure on $R$ such that the integral $\int_{-\infty}^{\infty} \Omega(x \sqrt{\lambda}) d \gamma(\lambda)$ converges absolutely for every $0 \leq x<a$, if and only if $f$ is positive definite on $[0, a)$.

The measure $\gamma$ may be chosen such that supp $\gamma \subset[0, \infty)((-\infty, 0])$ if and only if in addition to $f \in P_{\nu}[0, a)$

$$
\iint f(x, y)(-\Delta \phi)(x) \overline{\phi(y)} d \mu(x) d \mu(y) \geq 0 \quad(\leq 0)
$$

for all $\phi \in C_{0}^{\infty}(0, a / 2)$. Here $\Delta \phi(x)=\phi^{\prime \prime}(x)+(2 \nu / x) \phi^{\prime}(x)$.

Proof. If $f$ has an integral representation of the form (19), then, clearly, $f$ is locally essentially bounded on $[0, a)$. Further, by Proposition 2, Fubini's theorem and (17)

$$
\begin{aligned}
f(x, y) & =\int_{-\infty}^{\infty} \int_{0}^{\infty} \Omega(z \sqrt{\lambda}) D(x, y, z) d \mu(z) d y(\lambda) \\
& =\int_{-\infty}^{\infty} \Omega(x \sqrt{\lambda}) \Omega(y \sqrt{\lambda}) d y(\lambda) \quad \text { for } 0<x, y<a / 2 .
\end{aligned}
$$

Hence

$$
\sum_{i=1}^{n} \sum_{j=1}^{n} \alpha_{i} \bar{\alpha}_{j} f\left(x_{i}, x_{j}\right)=\int_{-\infty}^{\infty}\left|\sum_{i=1}^{n} \alpha_{i} \Omega\left(x_{i} \sqrt{\lambda}\right)\right|^{2} d \gamma(\lambda) \geq 0
$$


and $f \in P_{\nu}[0, a)$.

Conversely, suppose that $f \in P_{\nu}[0, a)$. Let $\mathcal{D}(-a / 2, a / 2)$ be the space of even complex valued $C^{\infty}$-functions on $(-a / 2, a / 2)$ with compact support.

$\mathfrak{D}^{\#}(-a / 2, a / 2)$ is a vector subspace of $\mathfrak{D}(-a / 2, a / 2)$ (the usual space of test functions on $(-a / 2, a / 2)$ of distribution theory with the Schwartz topology). We endow $\mathfrak{D}^{\sharp}(-a / 2, a / 2)$ with the topology induced by $\mathscr{T}(-a / 2, a / 2)$ and set $E=$ $\mathscr{D}^{\#}(-a / 2, a / 2) . E$ is a vector subspace of the nuclear space $\mathscr{D}(-a / 2, a / 2)$ and therefore is nuclear. Clearly $E$ is separable. On $E$ we define a pseudo-inner product by setting

$$
(\phi, \psi)=\int_{0}^{a / 2} \int_{0}^{a / 2} f(x, y) \phi(x) \overline{\psi(y)} d \mu(x) d \mu(y)
$$

for $\phi$ and $\psi$ in $E$. $(\phi, \phi) \geq 0$ since $f \in P_{\nu}[0, a)$.

If $\phi \in E$ then $\phi^{\prime}(0)=0$ since $\phi$ is even. Therefore $\phi^{\prime}(x)=x \chi_{\phi}(x)$ for all $x$, where $\chi_{\phi} \in E$. Clearly

$$
\frac{\phi^{\prime}(x)}{x} \rightarrow \phi^{\prime \prime}(0) \text { as } x \rightarrow 0 .
$$

Thus, $\chi_{\phi}(0)=\phi^{\prime \prime}(0)$ and $\chi_{\phi}$ is uniquely determined by $\phi$.

On $E$ we define the operator $A \phi=-\phi^{\prime \prime}-2 \nu \chi_{\phi}$; i.e. $(A \phi)(x)=-\phi^{\prime \prime}(x)-$ $(2 \nu / x) \phi^{\prime}(x)$ if $x \neq 0$ and $-\phi^{\prime \prime}(0)-2 \nu \phi^{\prime \prime}(0)=-(1+2 \nu) \phi^{\prime \prime}(0)$ if $x=0$. Clearly $A$ is a linear operator in $E$. Furthermore, $A$ is continuous for

$$
\left|\chi_{\phi}^{(n-1)}(x)\right| \leq k_{n} \sup _{|t| \leq|x|}\left|\phi^{(n+1)}(t)\right| \quad \text { for } n=1,2,3, \ldots
$$

and all $x$, where $k_{n}$ is a constant independent of $\phi$. (In fact $k_{n}=1 / n$ is the smallest possible constant for which the above inequality holds for all $\phi$. The verification of this is left to the reader.)

From (21) follows that if $\left(\phi_{n}\right)$ is a sequence in $E$ which converges to 0 then $\chi_{\phi_{n}} \rightarrow 0$ in $E$, whence $A$ is continuous.

Next we show that $A$ is symmetric relative to the pseudo-inner product $(\phi, \psi)$; i.e., $(A \phi, \psi)=(\phi, A \psi)$ for $\phi$ and $\psi$ in $E$. To see this we first show that

$$
(A \phi) \hat{(} t)=t^{2} \hat{\phi}(t) \text { for } \phi \in E \text { and all complex } t \text {. }
$$

Indeed, if $K=\left[2^{\nu-1 / 2} \Gamma(\nu+1 / 2)\right]^{-1}$

$$
\begin{aligned}
d \mu(x) & =K x^{2 \nu} d x, \\
(A \phi)(x) & =-x^{-2 \nu} d\left(x^{2 \nu} \phi^{\prime}(x)\right) / d x \quad \text { if } x \neq 0,
\end{aligned}
$$

and therefore 


$$
\begin{aligned}
-(A \phi) \hat{(}(t)=K \int_{0}^{\infty} \Omega(t x)\left(x^{2 \nu} \phi^{\prime}(x)\right)^{\prime} d x=-K t \int_{0}^{\infty} \Omega^{\prime}(t x) x^{2 \nu} \phi^{\prime}(x) d x \\
\quad=K t \int_{0}^{\infty}\left[x^{2 \nu} t \Omega^{\prime \prime}(t x)+2 \nu x^{2 \nu-1} \Omega^{\prime}(t x)\right] \phi(x) d x=t \int_{0}^{\infty}\left[t \Omega^{\prime \prime}(t x)+\frac{2 \nu}{x} \Omega^{\prime}(t x)\right] d \mu(x)
\end{aligned}
$$

by successive integration by parts. But Bessel's differential equation for $J_{\nu-1 / 2}(x)$ implies that $\Omega^{\prime \prime}(t x)+(2 \nu / t x) \Omega^{\prime}(t x)=-\Omega(t x)$ for all complex $t$ and $x$ (if we set $\left.\Omega^{\prime}(z) /\left.z\right|_{z=0}=\Omega^{\prime \prime}(0)\right)$. Therefore

$$
(A \phi) \hat{(}(t)=t^{2} \int_{0}^{\infty} \Omega(t x) \phi(x) d \mu(x)=t^{2} \hat{\phi}(t)
$$

for all $t$.

From (22) follows that

$$
(A \phi \times \bar{\psi}) \hat{(}(t)=t^{2} \hat{\phi}(t) \overline{\hat{\psi}}(t)=\hat{\phi}(t) \overline{t^{2} \hat{\psi}(t)}=(\phi \times \overline{A \phi})(t)
$$

whence $A \phi \times \bar{\psi}=\phi \times \overline{A \psi}$. But

$$
(\phi, \psi)=\int_{0}^{a / 2} \int_{0}^{a / 2} f(x, y) \phi(x) \overline{\psi(y)} d \mu(x) d \mu(y)=\int_{0}^{a} f(x)(\phi \times \bar{\psi})(x) d \mu(x)
$$

by Fibini's the orem. Therefore

$$
(A \phi, \psi)=\int_{0}^{\infty} f(x)(A \phi \times \bar{\psi})(x) d \mu(x)=\int_{0}^{\infty} f(x)(\phi \times \overline{A \psi})(x) d \mu(x)=(\phi, A \psi)
$$

for $\phi$ and $\psi$ in $E$.

In $E$ we introduce the involution $\phi^{*}(x)=\overline{\phi(x)}$. Clearly $A \phi^{*}=(A \phi)^{*}$ and $\left(\phi^{*}, \psi^{*}\right)=(\psi, \phi)$ for all $\phi$ and $\psi$ in $E$. All conditions of the theorem of expansion into generalized eigenvectors [4, Theorem 1] are satisfied. Hence there exists a bounded, positive Radon measure $\rho$ on $R$, for every $\lambda \in R$ a sequence of generalized eigenvectors $e_{k}^{\prime}(\lambda) \in E^{\prime}, 1 \leq k<d(\lambda)+1$, of $A$ (i.e. $\left\langle e_{k}^{\prime}(\lambda), A \phi\right\rangle$ $=\lambda\left\langle e_{k}^{\prime}(\lambda), \phi\right\rangle$ for all $\phi \in E$ and $\left.1 \leq k<d(\lambda)+1\right)$ such that

$$
(\phi, \psi)=\int_{-\infty}^{\infty} \sum_{k=1}^{d(\lambda)}\left\langle e_{k}^{\prime}(\lambda), \phi\right\rangle\left\langle\overline{e_{k}^{\prime}(\lambda)}, \bar{\psi}\right\rangle d \rho(\lambda)
$$

for $\phi, \psi \in E$. Here $d(\lambda)$ is a $\rho$-measurable function with values in $\{1,2,3, \ldots, \infty\}$ and there exists a $\rho$-null set $N_{0}$ such that $e_{k}^{\prime}(\lambda), 1 \leq k<d(\lambda)+1$, are linearly independent if $\lambda \notin N_{0}$. Furthermore supp $\rho \subset[0, \infty)((-\infty, 0])$ if $(20)$ holds. For details see loc. cit.

Let $G=(-a / 2, a / 2)$. Since $e_{k}^{\prime}(\lambda) \in \mathscr{D}^{\sharp}(G)^{\prime}$ there exists a unique even distribution $e_{k}(\lambda)$ on $G$ (i.e. $e_{k}(\lambda) \in \mathscr{D}^{\prime}(G)$ and $\left\langle e_{k}(\lambda), \phi\right\rangle=\left\langle e_{k}(\lambda), \check{\phi}\right\rangle$ for $\phi \in \mathscr{D}(G)$; here $\check{\phi}(x)=\phi(-x)$ ) whose restriction to $E$ is $e_{k}^{\prime}(\lambda), 1 \leq k<d(\lambda)+1, \lambda \in R$. See [4, Proposition 1].

If $\phi \in \mathscr{D}(G)$ we set $\phi^{\#}=1 / 2(\phi+\check{\phi}) ; \phi^{\#} \in E$. If now $\phi \in \mathscr{D}(G)$ and $\operatorname{supp} \phi \subset$ $G-\{0\}$, then clearly $-\Delta \phi=A \phi$ and $(-\Delta \phi)^{\#}=-\Delta \phi^{\#}$ since $\Delta$ is invariant 
under reflection $\phi \rightarrow \check{\phi}$, supp $\phi \subset R-\{0\}$. Hence, for all $\phi \in \mathscr{D}(G)$, supp $\phi \subset$ $G-\{0\}$,

$$
\left\langle e_{k}(\lambda),-\Delta \phi\right\rangle=\left\langle e_{k}(\lambda),(-\Delta \phi)^{\#}\right\rangle=\left\langle e_{k}(\lambda),-\Delta \phi^{\#}\right\rangle=\lambda\left\langle e_{k}(\lambda), \phi^{\#}\right\rangle=\lambda\left\langle e_{k}(\lambda), \phi\right\rangle
$$

since $e_{k}(\lambda)$ is even. Thus, the distribution $e_{k}(\lambda)$ satisfies the differential equation

$$
-{ }^{t} \Delta e_{k}(\lambda)=\lambda e_{k}(\lambda) \text { in } G-\{0\},
$$

$1 \leq k<d(\lambda)+1, \lambda \in R$. ' $\Delta$ denotes the transpose of $\Delta$,

$$
\left({ }^{t} \Delta \phi\right)(x)=\phi^{\prime \prime}(x)-\left(\frac{2 \nu}{x} \phi(x)\right)^{\prime}=\phi^{\prime \prime}(x)-\frac{2 \nu}{x} \phi^{\prime}(x)+\frac{2 \nu}{x^{2}} \phi(x),
$$

$\phi \in \mathscr{D}(G)$, supp $\phi \subset G-\{0\}$. It follows that the distribution $e_{k}(\lambda)$ is an even $C^{\infty}$. function $e_{k}(\lambda, x)$ in $G-\{0\}$ which satisfies the differential equation (23) [7, Theorem IX, p. 128] in the classical sense in $G-\{0\}$. In fact $e_{k}(\lambda, x)$ is an analytic function in $x, 0<|x|<a / 2$, since the coefficients of ${ }^{t} \Delta$ are analytic in $0<|x|$.

To find the general even solution of the differential equation (23),

$$
-{ }^{t} \Delta e=-e^{\prime \prime}+\left(\frac{2 \nu}{x} e\right)^{\prime}=\lambda e, \quad 0<|x|,
$$

set $e=|x|^{2 \nu} y$. Then by a simple computation

$$
-y^{\prime \prime}-\frac{2 \nu}{x} y^{\prime}=\lambda y, \quad x \neq 0 .
$$

The general even solution of this equation is, if $\lambda=0$,

$$
y(x)=\left\{\begin{array}{ll}
a_{1}|x|^{-2 \nu+1}+a_{2} & \text { if } \nu \neq 1 / 2, \\
a_{1} \log |x|+a_{2} & \text { if } \nu=1 / 2,
\end{array} \quad a_{1} \text { and } a_{2}\right. \text { are arbitrary constants. }
$$

If $\lambda \neq 0$,

$$
y(x)=|x|^{-(\nu-1 / 2)} J(\sqrt{\lambda}|x|), \quad x \neq 0,
$$

where $J(z)$ is the general solution of the Bessel equation

$$
z^{2} J^{\prime \prime}(z)+z J^{\prime}(z)+\left[z^{2}-(\nu-1 / 2)^{2}\right] J(z)=0, \quad z \neq 0 .
$$

The simple computation is left to the reader. Therefore

$$
y(x)=b_{1}|x|^{-(\nu-1 / 2)} J_{\nu-1 / 2}(\sqrt{\lambda}|x|)+b_{2}|x|^{-(\nu-1 / 2)} N_{\nu-1 / 2}(\sqrt{\lambda}|x|),
$$

$x \neq 0$, where $b_{1}$ and $b_{2}$ are arbitrary constants and $J_{\nu-1 / 2}$ is Bessel's function of index $\nu-1 / 2$ and $N_{\nu-1 / 2}$ is Neumann's function (or Bessel's function of the 2 nd kind) of index $\nu-1 / 2$.

Thus, 


$$
e_{k}(\lambda, x)= \begin{cases}C(0)|x|^{2 \nu}+D(0)|x|, & 0<|x|<a / 2, \text { if } \lambda=0, \nu=1 / 2, \\ C(0)|x|^{2 \nu}+D(0)|x|^{2 \nu} \log |x|, & 0<|x|<a / 2, \text { if } \lambda=0, \nu \neq 1 / 2,\end{cases}
$$

and

$$
e_{k}(\lambda, x)=C(\lambda)|x|^{2 \nu} \Omega(\sqrt{\lambda} x)+D(\lambda)|x|^{\nu+1 / 2} N_{\nu-1 / 2}(\sqrt{\lambda}|x|), \quad 0<|x|<a / 2, \text { if } \lambda \neq 0,
$$

where $C(\lambda)$ and $D(\lambda)$ are constants. Since $\Omega(0)=1$ we have in all cases

$$
e_{k}(\lambda, x)=C(\lambda)|x|^{2 \nu} \Omega(\sqrt{\lambda} x)+D(\lambda)|x|^{2 \nu} \theta_{\lambda}(x), \quad 0<|x|<a / 2,
$$

where $\theta_{0}(x)=|x|^{1-2 \nu}$ if $\nu \neq 1 / 2$ and $\log |x|$ if $\nu=1 / 2$ and $\theta_{\lambda}(x)=$ $|x|^{-(\nu-1 / 2)} N_{\nu-1 / 2}(\sqrt{\lambda}|x|)$ if $\lambda \neq 0$.

The function $|x|^{2 \nu} \theta_{\lambda}(x)$ is locally integrable at $x=0$ and therefore $e_{k}(\lambda, x)$ defines a distribution in $G$. Since $e_{k}(\lambda)=e_{k}(\lambda, x)$ (as distributions) in $G-\{0\}$, $e_{k}(\lambda)=e_{k}(\lambda, x)$ is a distribution in $G$ with support in $\{0\}$. Therefore

$$
e_{k}(\lambda)=e_{k}(\lambda, x)+\sum_{k=0}^{n} c_{k}(\lambda) \delta^{(k)}
$$

where $c_{k}(\lambda), k=0,1, \ldots, n$, are constants and $\delta$ is the Dirac delta measure. Since $e_{k}(\lambda)$ and $e_{k}(\lambda, x)$ are even distributions in $G$,

$$
\sum_{k=0}^{n} c_{k}(\lambda) \delta^{(k)} \text { is an even distribution. }
$$

Thus,

$$
\sum_{k=0}^{n} c_{k}(\lambda) \phi^{(k)}(0)=\sum_{k=0}^{n}(-1)^{k} c_{k}(\lambda) \phi^{(k)}(0)
$$

for all $\phi \in \mathscr{D}(G)$. It follows that $c_{k}(\lambda)=0$ for $k$ odd. Therefore

$$
e_{k}(\lambda)=e_{k}(\lambda, x)+\sum_{k=0}^{m} c_{2 k}(\lambda) \delta^{(2 k)} .
$$

We shall show that $e_{k}(\lambda)=c(\lambda)|x|^{2 \nu} \Omega(\sqrt{\lambda} x), \lambda \in R$. To prove this we first show that

$$
\left\langle e_{k}(\lambda, x), A \phi\right\rangle=\lambda\left\langle e_{k}(\lambda, x), \phi\right\rangle-2 \phi(0) \lim _{\epsilon \rightarrow 0_{+}} D(\lambda) \epsilon^{2 \nu} \theta^{\prime}(\epsilon)
$$

for every $\phi \in E$. Indeed, $e_{k}(\lambda, x)=|x|^{2 \nu} y(x)$, where $y$ satisfies (24). Therefore, if $\phi \in E$

$$
\begin{aligned}
\left\langle e_{k}(\lambda, x), A \phi\right\rangle & =2 \int_{0}^{a / 2} x^{2 \nu} y(x)(A \phi)(x) d x=-2 \int_{0}^{a / 2} y(x)\left(x^{2 \nu} \phi^{\prime}(x)\right)^{\prime} d x \\
& =2 \int_{0}^{a / 2} x^{2 \nu} y^{\prime}(x) \phi^{\prime}(x) d x, \text { for } \phi^{\prime}(0)=0
\end{aligned}
$$


and $\lim _{\epsilon \rightarrow 0+} \epsilon^{2 \nu} y(\epsilon)=\lim _{\epsilon \rightarrow 0+} e_{k}(\lambda, \epsilon)$ exists (in fact this limit is 0 as is easily verified). Therefore

$$
\begin{aligned}
\left\langle e_{k}(\lambda, x), A \phi\right\rangle & =2 \int_{0}^{a / 2}\left(x^{2 \nu} y^{\prime}(y)\right) \phi^{\prime}(x) d x=2 \lim _{\epsilon \rightarrow 0+} \int_{\epsilon}^{a / 2}\left(x^{2 \nu} y^{\prime}(x)\right) \phi^{\prime}(x) d x \\
& =-2 \lim _{\epsilon \rightarrow 0_{+}} x^{2 \nu} y^{\prime}(x) \phi(x)-2 \int_{0}^{a / 2}\left(x^{2 \nu} y^{\prime}(x)\right)^{\prime} \phi(x) d x \\
& =-2 \phi(0) \lim _{\epsilon \rightarrow 0+} \epsilon^{2 \nu} y^{\prime}(\epsilon)+2 \lambda \int_{0}^{a / 2}\left(x^{2 \nu} y(x)\right) \phi(x) d x \\
& =\lambda\left\langle e_{k}(\lambda, x), \phi\right\rangle-2 \phi(0) \lim _{\epsilon \rightarrow 0_{+}} D(\lambda) \epsilon^{2 \nu} \theta_{\lambda}^{\prime}(\epsilon)
\end{aligned}
$$

for $y(x)=C(\lambda) \Omega(\sqrt{\lambda} x)+D(\lambda) \theta_{\lambda}(x)$ by $(25)$ and

$$
\left.\frac{d \Omega(\sqrt{\lambda} x)}{d x}\right|_{x=0}=\sqrt{\lambda} \Omega^{\prime}(0)=0
$$

Now,

$$
\left\langle e_{k}(\lambda), A \phi\right\rangle=\lambda\left\langle e_{k}(\lambda), \phi\right\rangle \text { for all } \phi \in E,
$$

$\lambda \in k$ and $1 \leq k<d(\lambda)+1$. Therefore by (26)

$$
\left\langle e_{k}(\lambda, x), A \phi\right\rangle+\sum_{k=0}^{m} c_{2 k}(\lambda)(A \phi)^{(2 k)}(0)=\lambda\left\langle e_{k}(\lambda, x), \phi\right\rangle+\lambda \sum_{k=0}^{m} c_{2 k}(\lambda) \phi^{(2 k)}(0) .
$$

Hence, by (27),

(28) $-2 \phi(0) \lim _{\epsilon \rightarrow 0+} D(\lambda) \epsilon^{2 \nu} \theta_{\lambda}^{\prime}(\epsilon)+\sum_{k=0}^{m} c_{2 k}(\lambda)(A \phi)^{(2 k)}(0)-\lambda \sum_{k=0}^{m} c_{2 k}(\lambda) \phi^{(2 k)}(0)=0$.

Since (28) holds for all $\phi \in E$, the coefficients of $\phi(0), \phi^{\prime \prime}(0), \ldots, \phi^{(2 m+2)}(0)$ in $(28)$ are all 0 . Now $(A \phi)(0)=-(1+2 \nu) \phi^{\prime \prime}(0)$, and by a simple computation

$$
(A \phi)^{(2 k)}(0)=-\left(1+\frac{2 \nu}{2 k+1}\right) \phi^{(2 k+2)}(0), \quad k=0,1,2, \ldots
$$

Therefore the coefficient of $\phi^{(2 m+2)}(0)$,

$$
-c_{2 m}(\lambda)\left(1+\frac{2 \nu}{2 m+1}\right)=0
$$

whence, $c_{2 m}(\lambda)=0$. Continuing this way we see that $c_{0}(\lambda)=c_{2}(\lambda)=\cdots=$ $c_{2 m}(\lambda)=0$ for every $\lambda \in R$. Therefore $e_{k}(\lambda)=e_{k}(\lambda, x)$ and

$$
\lim _{\epsilon \rightarrow 0+} D(\lambda) \epsilon^{2 \nu} \theta_{\lambda}^{\prime}(\epsilon)=0, \quad \lambda \in R
$$


But $\lim _{\epsilon \rightarrow 0_{+}} \epsilon^{2 \nu} \theta_{\lambda}^{\prime}(\epsilon) \neq 0$. The reader will verify that when $\lambda=0, \lim _{\epsilon \rightarrow 0_{+}} e^{2 \nu} \theta_{0}^{\prime}(\epsilon)=1$ if $\nu=1 / 2,(1-2 \nu)$ if $\nu \neq 1 / 2$ and

$$
\lim _{\epsilon \rightarrow 0_{+}} \epsilon^{2 \nu} \theta^{\prime}(\theta)=\frac{2^{(\nu+1 / 2)}(\sqrt{\lambda})^{(1 / 2-\nu)} \Gamma(\nu+1 / 2)}{\pi} \text { if } \lambda \neq 0 .
$$

It follows therefore from (29) that $D(\lambda)=0$ for all $\lambda \in R$. Hence

$$
e_{k}(\lambda)=C(\lambda)|x|^{2 \nu} \Omega(\sqrt{\lambda} x), \quad \lambda \in R
$$

Therefore $d(\lambda)=1 \quad \rho$-a.e. and

$$
\left\langle e_{1}(\lambda), \phi\right\rangle\left\langle\overline{\left.e_{1}(\lambda), \psi\right\rangle}=\left|\frac{2 C(\lambda)}{K}\right|^{2} \int_{0}^{a / 2} \int_{0}^{a / 2} \Omega(x \sqrt{\lambda}) \Omega(y \sqrt{\lambda}) \phi(x) \overline{\psi(y)} d \mu(x) d \mu(y)\right.
$$

belongs to $L^{1}(\rho)$ for all $\phi$ and $\psi$ in $E$. Therefore $\lambda \rightarrow 4 K^{-2}|C(\lambda)|^{2}$ is $\rho$-measurable and if we set $d y(\lambda)=4 K^{-2}|C(\lambda)|^{2} d \rho(\lambda)$,

$$
\begin{aligned}
(\phi, \psi) & =\int_{0}^{a / 2} \int_{0}^{a / 2} f(x, y) \phi(x) \overline{\psi(y)} d \mu(x) d \mu(y) \\
& =\int_{-\infty}^{\infty} \int_{0}^{a / 2} \int_{0}^{a / 2} \Omega(x \sqrt{\lambda}) \Omega(y \sqrt{\lambda}) \phi(x) \overline{\psi(y)} d \mu(x) d \mu(y) d \gamma(\lambda) .
\end{aligned}
$$

We now proceed mutatis mutandis as in the proof of [4, Theorem 31.

If $0<x_{0}<a / 2$ is an arbitrary but fixed point, choose a sequence $\left(\phi_{n}\right)$ in $E$ such that $\phi_{n} \geq 0, \int_{0}^{a / 2} \phi_{n} d \mu=1$ and supp $\phi_{n} \subset\left\{x \in R|||x|-x_{0} \mid<1 / n\right\}$. Then

$$
\int_{0}^{a / 2} \Omega(x \sqrt{\lambda}) \phi_{n}(x) d x \rightarrow \Omega\left(x_{0} \sqrt{\lambda}\right) \text { as } n \rightarrow \infty
$$

and therefore upon substitution of $\phi_{n}$ for $\phi$ and $\psi$ in (30) and letting $n \rightarrow \infty$ we obtain by Fatou's lemma and (ii) of Proposition 2

$$
\int_{-\infty}^{\infty}\left(\Omega\left(x_{0} \sqrt{\lambda}\right)\right)^{2} d y(\lambda) \leq \frac{\lim }{n \rightarrow \infty} \int_{0}^{a / 2} \int_{0}^{a / 2} f(x, y) \phi_{n}(x) \phi_{n}(y) d \mu(x) d \mu(y)=f\left(x_{0}, x_{0}\right) .
$$

Therefore if $0<x, y<a / 2$,

$$
\begin{aligned}
\int_{-\infty}^{\infty}|\Omega(x \sqrt{\lambda}) \Omega(y \sqrt{\lambda})| d y(\lambda) & \leq\left(\int_{-\infty}^{\infty}(\Omega(x \sqrt{\lambda}))^{2} d y(\lambda)\right)^{1 / 2}\left(\int_{-\infty}^{\infty}(\Omega(y \sqrt{\lambda}))^{2} d y(\lambda)\right)^{1 / 2} \\
& \leq f(x, x)^{1 / 2} f(y, y)^{1 / 2}
\end{aligned}
$$

Now

$$
f(x, x)=\int_{0}^{a} f(z) D(x, x, z) d \mu(z) \leq \underset{0 \leq z \leq 2 x}{\text { ess sup }}|f(z)|<\infty, \quad 0<x<a / 2
$$

and therefore by Fatou's lemma if $x_{n} \rightarrow 0,0<x_{n}<a / 2$,

$$
\int_{-\infty}^{\infty} d y(\lambda) \leq \lim _{n \rightarrow \infty} f\left(x_{n}, x_{n}\right)=\lim _{x \rightarrow 0_{+}} f(x, x)<\infty .
$$

Thus $\gamma$ is a bounded positive Radon measure.

Next we show that 


$$
f(x, y)=\int_{-\infty}^{\infty} \Omega(x \sqrt{\lambda}) \Omega(y \sqrt{\lambda}) d y(\lambda), \quad 0<x, y<a / 2
$$

To see this we first note that

$$
\begin{aligned}
& \left.\int_{-\infty}^{\infty} \int_{0}^{a / 2} \int_{0}^{a / 2} \mid \Omega(x \sqrt{\lambda}) \Omega(y \sqrt{\lambda}) \phi(x) \overline{\psi(y}\right) \mid d \mu(x) d \mu(y) d y(\lambda) \\
& \leq\left(\int_{-\infty}^{\infty} \int_{0}^{a / 2}|\Omega(x \sqrt{\lambda}) \phi(x)|^{2} d \mu(x) d y(\lambda)\right)^{1 / 2}\left(\int_{-\infty}^{\infty} \int_{0}^{a / 2}|\Omega(y \sqrt{ } \lambda) \psi(y)|^{2} d \mu(y) d y(\lambda)\right)^{1 / 2} \\
& =(\phi, \phi)(\psi, \psi)<\infty \text { for all } \phi \text { and } \psi \text { in } E \text {. }
\end{aligned}
$$

Therefore from (30) by Fubini's theorem

(33) $\int_{0}^{a / 2} \int_{0}^{a / 2} f(x, y) \phi(x) \overline{\psi(y)} d \mu(x) d \mu(y)=\int_{0}^{a / 2} \int_{0}^{a / 2} K(x, y) \phi(x) \overline{\psi(y)} d \mu(x) d \mu(y)$

for all $\phi$ and $\psi$ in $E$, where

$$
K(x, y)=\int_{-\infty}^{\infty} \Omega(x \sqrt{\lambda}) \Omega(y \sqrt{\lambda}) d \gamma(\lambda), \quad 0 \leq x, y<a / 2 .
$$

$K(x, y)$ is continuous on $[0, a / 2) \times[0, a / 2)$ for

$$
(x, y) \rightarrow \int_{0}^{\infty} \Omega(x \sqrt{\lambda}) \Omega(y \sqrt{\lambda}) d y(\lambda)
$$

is continuous on $R \times R$ by the Lebesgue bounded convergence theorem since $|\Omega(z)| \leq 1$ for $0 \leq z<\infty$ and

$$
(x, y) \rightarrow \int_{-\infty}^{0} \Omega(x \sqrt{\lambda}) \Omega(y \sqrt{\lambda}) d y(\lambda)
$$

is continuous on $[0, a / 2) \times[0, a / 2)$ by the Lebesgue dominated convergence theorem because $1 \leq \Omega(i x) \leq \Omega\left(i x_{1}\right)$ for $0 \leq x \leq x_{1}<\infty$ and (31) holds.

From (33) and the fact that $f(x, y)$ and $K(x, y)$ are both continuous on $(0, a / 2) \times(0, a / 2)$ follows that

$$
f(x, y)=K(x, y) \quad \text { for } 0<x, y<a / 2 \text {, i.e. (32). }
$$

Next we define the function $k(x), 0 \leq x<a$, by $k(x)=\int_{-\infty}^{\infty} \Omega(x \sqrt{\lambda}) d y(\lambda)$. This integral converges absolutely for $0 \leq x<a$. Indeed, if $0<x<a / 2$ then

$$
\begin{aligned}
f(x, x) & =\int_{-\infty}^{\infty}(\Omega(x \sqrt{\lambda}))^{2} d y(\lambda)=\int_{-\infty}^{\infty} \int_{0}^{a} \Omega(\sqrt{\lambda} z) D(x, x, z) d \mu(z) d \gamma(\lambda) \\
& =\int_{0}^{a} \int_{-\infty}^{\infty} \Omega(\sqrt{\lambda} z) D(x, x, z) d \gamma(\lambda) d \mu(z) .
\end{aligned}
$$

The change of order of integration is justified for

$$
\int_{-\infty}^{0} \int_{0}^{a} \Omega(\sqrt{\lambda} z) D(x, x, z) d \mu(z) d y(\lambda)=\int_{0}^{a} \int_{-\infty}^{0} \Omega(\sqrt{\lambda} z) D(x, x, z) d \gamma(\lambda) d \mu(z)
$$

since the integrand is nonnegative. Further

$$
\begin{aligned}
\int_{0}^{\infty} \int_{0}^{a}|\Omega(\sqrt{\lambda} z)| D(x, x, z) d \mu(z) d \gamma(\lambda) \\
\quad \leq \int_{0}^{\infty} \int_{0}^{a} D(x, x, z) d \mu(z) d \gamma(\lambda)=\int_{0}^{\infty} d \gamma(\lambda) \leq \int_{-\infty}^{\infty} d \gamma(\lambda)<\infty .
\end{aligned}
$$


Therefore Fubini's theorem applies.

From (34) follows by Fubini's theorem that

$$
\int_{-\infty}^{\infty}|\Omega(\sqrt{\lambda} z)| D(x, x, z) d y(\lambda)<\infty, \quad \mu \text {-a.e. }
$$

Hence, since $D(x, x, z) \neq 0$ for $0<z<2 x$,

$$
\int_{-\infty}^{\infty}|\Omega(\sqrt{\lambda} z)| d y(\lambda)<\infty \text { a.e. for } 0<z<2 x .
$$

But $\int_{0}^{\infty}|\Omega(\sqrt{\lambda} z)| d y(\lambda)<\infty$ for all $0 \leq z<\infty$ and $x \rightarrow \Omega(\sqrt{\lambda} x)$ is monotonically increasing if $\lambda<0$. Therefore

$$
\int_{-\infty}^{\infty}|\Omega(\sqrt{\lambda} z)| d y(\lambda)<\infty \text { for all } 0 \leq z<2 x
$$

and since $0<x<a / 2$ was arbitrary

$$
\int_{-\infty}^{\infty}|\Omega(\sqrt{\lambda} z)| d y(\lambda)<\infty \text { for all } 0 \leq z<a .
$$

From what precedes also follows by the Lebesgue dominated convergence theorem that $k(x)$ is continuous on $0 \leq x<a$ and $f(x, y)$ is the associated function of $k$. Indeed

$$
\begin{aligned}
k(x, y) & =\int_{0}^{a} k(z) D(x, y, z) d \mu(z)=\int_{0}^{a} \int_{-\infty}^{\infty} \Omega(\sqrt{\lambda} z) D(x, y, z) d y(\lambda) d \mu(z) \\
& =\int_{-\infty}^{\infty} \int_{0}^{a} \Omega(\sqrt{\lambda} z) D(x, y, z) d \mu(z) d y(\lambda)=\int_{-\infty}^{\infty} \Omega(x \sqrt{\lambda}) \Omega(y \sqrt{\lambda}) d y(\lambda) \\
& =f(x, y) \text { for } 0<x, y<a / 2,
\end{aligned}
$$

by Fubini's theorem and (17). Therefore by Lemma 1

$$
f(x)=k(x) \text { a.e. for } 0 \leq x<a .
$$

That is,

$$
f(x)=\int_{-\infty}^{\infty} \Omega(x \sqrt{\lambda}) d \gamma(\lambda) \text { a.e. for } 0 \leq x<a
$$

Finally, if

$$
f(x)=\int_{-\infty}^{\infty} \Omega(x \sqrt{\lambda}) d y(\lambda) \text { a.e. for } 0 \leq x<a,
$$

where the integral converges absolutely for $0 \leq x<a$, and supp $y \subset$ $[0, \infty)((-\infty, 0])$, then for all $\phi \in E$

$$
\begin{aligned}
& \iint f(x, y)(-\Delta \phi)(x) \overline{\phi(y)} d \mu(x) d \mu(y) \\
& \quad=\iiint \int_{-\infty}^{\infty} \Omega(z \sqrt{\lambda}) D(x, y, z)(-\Delta \phi)(x) \overline{\phi(y)} d y(\lambda) d \mu(z) d \mu(x) d \mu(y) \\
& \quad=\int_{-\infty}^{\infty} \iiint \Omega(z \sqrt{\lambda}) D(x, y, z)(-\Delta \phi)(x) \overline{\phi(y)} d \mu(z) d \mu(x) d \mu(y) d y(\lambda) \\
& \left.\quad=\int_{-\infty}^{\infty}(-\Delta \phi) \hat{(} \sqrt{\lambda}\right) \bar{\phi}(\sqrt{\lambda}) d y(\lambda)=\int_{-\infty}^{\infty} \lambda|\hat{\phi}(\sqrt{\lambda})|^{2} d y(\lambda) \geq 0 \quad(\leq 0)
\end{aligned}
$$


by successive application of Fubini's theorem and (22). This completes the proof.

Corollary 1. If $f \in P_{\nu}[0, a)$ there exists a continuous function $k \in P_{\nu}[0, a)$ such that $f(x)=k(x)$ a.e.

Corollary 2. A continuous function $f$ on the interval $[0, a)(0<a \leq \infty)$ bas an integral representation of the form

$$
f(x)=\int_{-\infty}^{\infty} \Omega(x \sqrt{\lambda}) d y(\lambda) \text { for all } 0 \leq x<a,
$$

where $\gamma$ is a bounded, positive Radon measure on $R$, if and only if $f \in P_{\nu}[0, a)$.

Corollary 3. [1]. A function $f \in L^{\infty}[0, \infty)$ (relative to the Lebesgue measure) has an integral representation of the form

$$
f(x)=\int_{0}^{\infty} \Omega(x t) d y(t) \text { a.e. for } 0 \leq x<\infty,
$$

where $\gamma$ is a bounded, positive Radon measure on $[0, \infty)$, if and only if $f \epsilon$ $P_{\nu}[0, \infty)$.

Lemma 2. A continuous function $f \in P_{\nu}[0, a)$ is $[\nu]$-times continuously differentiable. See [6, Lemma 4, p. 8221.

Proof. If $f \in P_{\nu}[0, a)$ is continuous

$$
f(x)=\int_{-\infty}^{\infty} \Omega(x \sqrt{\lambda}) d y(\lambda), \quad 0 \leq x<a,
$$

by Corollary 2. Now $x \rightarrow \int_{-\infty}^{0} \Omega(x \sqrt{\lambda}) d y(\lambda)$ is an analytic function on $-a<x<a$ for

$$
\Omega(x \sqrt{\lambda})=\sum_{k=0}^{\infty}(-1)^{k} c_{k}(x \sqrt{\lambda})^{2 k}=\sum_{k=0}^{\infty} c_{k} x^{2 k}(-\lambda)^{k},
$$

where the coefficients $c_{k}>0, k=0,1,2, \ldots$ Therefore

$$
\int_{-\infty}^{0} \Omega(x \sqrt{\lambda}) d y(\lambda)=\sum_{k=0}^{\infty}\left(c_{k} \int_{-\infty}^{0}(-\lambda)^{k} d y(\lambda)\right) x^{2 k}
$$

for $|x|<a$.

Next we show that $g(x)=\int_{0}^{\infty} \Omega(x \sqrt{\lambda}) d y(\lambda)$ is [ $\left.\nu\right]$-times continuously differentiable on $R$. We proceed as in [6, Lemma 4., p. 822].

$$
\frac{\partial^{k} \Omega(x \sqrt{\lambda})}{\partial x^{k}}=\Omega^{(k)}(x \sqrt{\lambda})(\sqrt{\lambda})^{k} .
$$

From $\Omega(x)=c_{a^{x}} x^{-a} J(x)$, where $\alpha=\nu-1 / 2$ and $c_{a}$ is a positive constant, follows that

$$
c_{a}^{-1} \Omega^{(k)}(x)=J_{a}^{(k)}(x) x^{-a}-\left(\begin{array}{l}
k \\
1
\end{array}\right) a J_{a}^{(k-1)}(x) x^{-a-1}+\cdots \pm a(\alpha+1) \cdots(\alpha+k-1) J_{a}(x) x^{-a-k} .
$$


Hence, since $J_{a}^{(k)}(x)=O\left(x^{-1 / 2}\right)$, we have $\Omega^{(k)}(x)=O\left(x^{-a-1 / 2}\right)=O\left(x^{-\nu}\right)$. Therefore by (35)

$$
\frac{\partial^{k} \Omega(x \sqrt{\lambda})}{\partial x^{k}}=O\left((\sqrt{\lambda})^{k-\nu}\right) .
$$

It follows that if $k \leq \nu$, i.e. $k<[\nu]$, then

$$
g^{(k)}(x)=\int_{0}^{\infty} \frac{\partial^{k} \Omega(x \sqrt{\lambda})}{\partial x^{k}} d y(\lambda)
$$

for all $x \in R$.

Suppose $f$ is a function of class $C^{2}$ on the interval $[0, a)$, then by a simple computation

$$
\int_{0}^{a} f(x)(\Delta \phi)(x) d \mu(x)=\int_{0}^{a}(\Delta f)(x) \phi(x) d \mu(x)
$$

for all $\phi \in C_{0}^{\infty}(0, a)$. Indeed, since $d \mu(x)=K x^{2 \nu} d x$, where $K$ is a constant,

$$
\begin{aligned}
& \int_{0}^{a} f(x)(\Delta \phi)(x) d \mu(x)=K \int_{0}^{a} f(x)(\Delta \phi)(x) x^{2 \nu} d x \\
& =K \int_{0}^{a} f(x)\left(x^{2 \nu} \phi^{\prime}(x)\right)^{\prime} d x=-K \int_{0}^{a} f^{\prime}(x) x^{2 \nu} \phi^{\prime}(x) d x \\
& =K \int_{0}^{a} \phi(x)\left(x^{2 \nu} f^{\prime}(x)\right)^{\prime} d x=\int_{0}^{a}(\Delta f(x) \phi(x) d \mu(x) .
\end{aligned}
$$

From (36) follows that condition (20) of Theorem 2

$$
\begin{aligned}
& \iint f(x, y)(-\Delta \phi)(x) \overline{\phi(y)} d \mu(x) d \mu(y)=\int f(x)((-\Delta \phi) \times \bar{\phi})(x) d \mu(x) \\
& \quad=\int f(x)(-\Delta(\phi \times \bar{\phi}))(x) d \mu(x)=\int(-\Delta f)(x)(\phi \times \bar{\phi})(x) d \mu(x) \geq 0 \quad(\leq 0)
\end{aligned}
$$

for all $\phi \in C_{0}^{\infty}(0, a)$ is equivalent to $-\Delta f(\Delta f) \in P_{\nu}[0, a)$. Combining this with Lemma 2 we may restate the last part of Theorem 2 as follows.

Theorem 3. Suppose $\nu \geq 2$. A continuous function $f$ on $[0, a)(0<a \leq \infty)$ bas an integral representation of the form

$$
f(x)=\int_{0}^{\infty} \Omega(x t) d \beta(t), \quad\left(f(x)=\int_{0}^{\infty} \Omega(i x t) d \beta(t)\right)
$$

for $0 \leq x<a$, where $\beta$ is a bounded, positive Radon measure on $[0, \infty)$, if and only if $f$ and $-\Delta f(\Delta f)$ belong to $P_{\nu}[0, a)$.

We conclude this paper with the following theorem.

Theorem 4. $P_{\nu}[0, a) \supset P_{\mu}[0, a)$ if $\nu<\mu$ and a function $f \in P_{\infty}[0, a)=$ $\bigcap_{\nu>0} P_{\nu}[0, a)$ if and only if $f$ bas an integral representation of the form

$$
f(x)=\int_{-\infty}^{\infty} e^{x^{2} t} d \omega(t) \text { a.e. for } 0 \leq x<a
$$


where $\omega$ is a bounded, positive Radon measure on $R$ such that

$$
\int_{-\infty}^{\infty} e^{x^{2} t} d \omega(t)<\infty \text { for all } 0 \leq x<a .
$$

Proof. We start with the identity [8, p. 373]

$$
J_{a_{+} \beta+1}(z)=\frac{z^{a_{+}}}{2^{\alpha} \Gamma(\alpha+1)} \int_{0}^{\pi / 2} J_{\beta}(z \sin \theta)(\sin \theta)^{\beta+1}(\cos \theta)^{2 a_{+} 1} d \theta
$$

$\alpha>-1, \beta>-1, z$ any complex number. From it follows that if $\mu>\nu(>0)$

$$
\Omega_{2 \mu+1}(z)=\int_{0}^{\pi / 2} \Omega(z \sin \theta) d \sigma(\theta)
$$

for all complex numbers $z$. Here $\Omega=\Omega_{2 \nu_{+1}}$ and $\sigma$ is a bounded, positive Radon measure on $[0, \pi]$ (depending upon $\nu$ and $\mu$ ).

If now $f \in P_{\mu}[0, a), \nu<\mu$, then by Theorem $2 f$ has an integral representation

$$
f(x)=\int_{-\infty}^{\infty} \Omega_{2 \mu+1}(x \sqrt{\lambda}) d y(\lambda) \text { a.e. for } 0 \leq x<a,
$$

where $\gamma$ is a bounded, positive Radon measure on $[0, a)$ for which

$$
\int_{-\infty}^{\infty}\left|\Omega_{2 \mu+1}(x \sqrt{\lambda})\right| d y(\lambda)<\infty \text { for } 0 \leq x<a .
$$

Let $f(x, y), 0<x, y<a / 2$, be the associated function of $f$ relative to $\nu$, then

$$
\begin{aligned}
f(x, y) & =\int_{0}^{a} f(z) D(x, y, z) d \mu(z)=\int_{0}^{a} \int_{-\infty}^{\infty} \Omega_{2 \mu+1}(z \sqrt{\lambda}) D(x, y, z) d y(\lambda) d \mu(z) \\
& =\int_{0}^{a} \int_{-\infty}^{\infty} \int_{0}^{\pi / 2} \Omega(z \sqrt{\lambda} \sin \theta) D(x, y, z) d \sigma(\theta) d y(\lambda) d \mu(z) \\
& =\int_{-\infty}^{\infty} \int_{0}^{\pi / 2} \int_{0}^{a} \Omega(z \sqrt{\lambda} \sin \theta) D(x, y, z) d \mu(z) d \sigma(\theta) d y(\lambda) \\
& =\int_{-\infty}^{\infty} \int_{0}^{\pi / 2} \Omega(x \sqrt{\lambda} \sin \theta) \Omega(y \sqrt{\lambda} \sin \theta) d \sigma(\theta) d y(\lambda), \quad 0<x, y<a / 2,
\end{aligned}
$$

by (38), (37), Fubini's theorem and (17) in that order. From the last equality follows that $f \in P_{\nu}[0, a)$; i.e. $P_{\nu}[0, a) \supset P_{\mu}[0, a)$.

From what precedes follows that $P_{\infty}[0, a)=\bigcap P_{1 / 2(N-1)}[0, a)$ as $N$ ranges over the integers $2,3,4, \ldots$ In [4] we proved [loc. cit., Theorem 51 that a continuous function

$$
k \in P_{1 / 2(N-1)}[0, a) \text { for } N=2,3,4, \cdots
$$

if and only if $k$ has a representation of the form

$$
k(x)=\int_{-\infty}^{\infty} e^{x^{2} t} d \omega(t) \quad \text { for } 0 \leq x<a,
$$

where $\omega$ is a bounded, positive Radon measure on $R$. But to every $f \in P_{\nu}[0, a)$ there exists a (unique) continuous $k \in P_{\nu}[0, a)$ such that $f(x)=k(x)$ a.e., by 
Corollary 1. Hence, to every $f \in P_{\infty}[0, a)$ there exists a (unique) continuous $k \epsilon$ $P_{\infty}[0, a)$ such that $f(x)=k(x)$ a.e. Therefore, from what precedes, a function $f$ on $[0, a)$ belongs to $P_{\infty}[0, a)$ if and only if there exists a bounded, positive Radon measure $\omega$ on $R$ such that

$$
f(x)=\int_{-\infty}^{\infty} e^{x^{2} t} d \omega(t) \text { a.e. for } 0 \leq x<a .
$$

\section{REFERENCES}

1. F. M. Cholewinski, D. T. Haimo and A. E. Nussbaum, A necessary and sufficient condition for the representation of a function as a Hankel-Stieltjes transform, Studia Math. 36 (1970), 269-274. MR $42 \# 6535$.

2. I. I. Hirschman, Jr., Variation diminishing Hankel transforms, J. Analyse Math. 8 (1960/61), 307-336. MR 28 \#433.

3. A. E. Nussbaum, Radial exponentially convex functions, J. Analyse Math. 25 (1972), 277-288.

4. - Integral representation of functions and distributions positive definite relative to the orthogonal group, Trans. Amer. Math. Soc. 175 (1973), 355-387.

5. W. Rudin, An extension theorem for positive-definite functions, Duke Math. J. 37 (1970), 49-53. MR $40 \# 7722$.

6. I. J. Schoenberg, Metric spaces and completely monotone functions, Ann. of Math. (2) 39 (1938), 811-841.

7. L. Schwartz, Théorie des distributions. Tome I, Actualités Sci. Indust., no. 1091, Hermann, Paris, 1950. MR 12, 31.

8. G. N. Watson, A treatise on the theory of Bessel functions, 2nd ed., Cambridge Univ. Press, Cambridge; Macmillan, New York, 1944. MR 6, 64.

DEPARTMENT OF MATHEMATICS, WASHINGTON UNIVERSITY, ST. LOUIS, MISSOURI 63130 